\title{
SANS study of structures and deuterium incorporation into vegetative leaf stalks of deuterated Kale (Brassica oleracea)
}

Zhi Yang ${ }^{1}$, Sai Venkatesh Pingali, Hugh O'Neill', Sam Bhagia ${ }^{1}$, Barbara R. Evans ${ }^{2}$, Brian H. Davison ${ }^{3}$, Arthur Ragauskas 3,4

${ }^{1}$ Neutron Scattering Division, ${ }^{2}$ Chemical Sciences Division, ${ }^{3}$ Biosciences Division, Oak Ridge

National Laboratory, Oak Ridge Tennessee 37831

${ }^{4}$ Department of Chemical and Biomolecular Engineering, University of Tennessee, Knoxville,

Tennessee 37996

\section{Email Contact: yangz2@ornl.gov}

The unique capabilities of small angle neutron scattering (SANS) assisted by NMR and FTIR have been applied to reveal the detailed molecular structure of both natural lignocellulosic biomass and model cellulosic composites. In this study, these techniques are applied to examine the stem structure of a deuterated herbaceous dicotyledonous plant, kale, which exhibits a hierarchical structure that resembles that of wood in vascular architecture. In this study, partial deuteration was achieved through cultivating the kale plant in deuterated media to enhance contrast between component plant biopolymers. Cellulose microfibrils that solely contributes to SANS data in high$Q$ region $\left(Q>0.08 \AA^{-1}\right)$ is determined to scatter similar to $65 \% \mathrm{D}_{2} \mathrm{O}$ solvent ( $\mathrm{SLD} \sim 3.94 \mathrm{E}-06 \AA^{-2}$ ), indicating that approximately $50 \%$ of the available covalently bonded $\mathrm{H}$ atoms would be substituted by $\mathrm{D}$ atoms. In the low- $Q$ region, the features responsible for these power-law decay show a neutron sensitivity that matches a solvent of $48 \% \mathrm{D}_{2} \mathrm{O}\left(\mathrm{SLD} \sim 2.77 \mathrm{E}-06 \AA^{-2}\right.$ ). To result in an overall SLD that is lower than cellulose, the SLD of lignin and hemicellulose needs to be lower to off-set the high SLD of cellulose within cell wall. To confirm the deuterium incorporation in the cellulose component of deuterated kale stem, contrast variation SANS experiments have been carried out on extracted cellulose. The results demonstrated that the contrast matching points exhibit a nearly constant value of $\sim 70 \% \mathrm{D}_{2} \mathrm{O}$ over the whole $Q$ range, which is comparable to $65 \%$ $\mathrm{D}_{2} \mathrm{O}$ obtained for the cellulose component in the deuterated kale stem. Finally, this study revealed the hierarchical structures of deuterated kale stems and varying amounts of deuterium incorporation in the different cell wall component polymers. 